Journal of Engineering Sciences Faculty of Engineering Assiut University

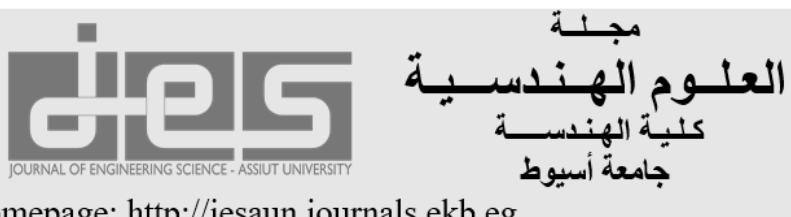

journal homepage: http://jesaun.journals.ekb.eg

\title{
MHD Natural Convection of grade three of non-Newtonian fluid flow between two Vertical Flat Plates through Porous Medium with heat source effect
}

Received 12 December 2021; Revised 21 January 2022; Accepted 22 January 2022

\section{Hussein Abd Allah Soliman ${ }^{1}$}

\section{Keywords}

MHD Natural convection, non-Newtonian fluid, Porous Medium, Heat source, Analytical and numerical solution

\begin{abstract}
Analytical and numerical solutions are investigated to study solution of the Magneto hydrodynamics (MHD) natural convection flow of grade three of a non- Newtonian fluid flow between two vertical flat plates through embedded in a porous medium and considering the effect heat source using Multi-step differential transform method and finite difference method. The system of coupled nonlinear ordinary differential equations are solved analytically using Multi-step differential transform method (MDTM) and numerically using finite difference method (FDM). The results of (MDTM), (FDM), and another analytical method are all in good agreement, demonstrating that these methods are capable of solving similar problems. Graphs and tables show the effect of various parameters on velocity and temperature. The current studies, as well as comparisons to previous findings, are presented in figures and tables. The study results showed that the analytical solution using Multi-step differential transform method and numerical solution using finite difference method agrees well with recent analytical and numerical solutions.
\end{abstract}

\section{Introduction}

Geothermal systems, heat exchangers, chemical catalytic reactors, fibre and granular insulation, packed beds, petroleum reservoirs, and nuclear waste dumps all use natural convection to transfer heat. Numerous [16] writers have investigated the flow of Newtonian and non-Newtonian fluids between two infinite parallel vertical plates, showing its importance. A non-Newtonian fluid's flow properties are not defined by a single constant value of viscosity.

The relationship between shear stress and strain rate in a Newtonian fluid is linear (It would pass past the origin if plotted), using the coefficient of viscosity as the proportionality constant. In a nonNewtonian fluid, the relationship between shear stress and strain rate is nonlinear and can even be time dependent. As a result, calculating a constant viscosity coefficient is impossible. This idea is more relevant for fluids with no time dependent behaviour, as it may be described as a proportion of shear stress to strain rate. Although viscosity is a typical way to describe a material's properties, it is often insufficient to describe a substance's material behaviour, especially in non-Newtonian fluids. They're best evaluated with a number of other rheological properties that link the relationships

${ }^{1}$ Department of Basic Science, Engineering Division, International Academy for Engineering and Media Science,

6th October, Egypt ( $\underline{\text { Hussein.AbdAllah.Soliman@iaems.edu.eg })}$ 
between the stress and strain rate tensors under various flow conditions, such as oscillatory shear or extensional flow, and are measured with rhinometries. Tensor-valued constitutive equations, which are common in the field of continuum mechanics [16], are excellent for studying the properties.

For a class of non-Newtonian fluids, Bruce and $\mathrm{Na}$ [1] investigated the natural convection problem between vertical flat plates. Other heat transfer problems using laminar natural convection have also been investigated ([2], [3] and [22]). On the other hand, Rajagopal [20] offered a thorough thermodynamic analysis of the constitutive functions. Ordinary or partial differential equations are used to model certain scientific problems and occurrences. Because analytical solutions are rarely applicable to these problems, special procedures must be used to solve these equations.

In recent years, there has been a lot of focus on newly discovered methods for constructing an analytic and numerical solution to equations ([21], [23]). The so-called tiny parameters are overly reliant on perturbation approaches[19]. There have been other different methods for solving nonlinear equations introduced, containing the Adomian decomposition technique (ADM) ([11], [12]), Hamiltonian Approach ([5], [17]), Exp-Function approach ([13], [14]) and Homotopy perturbation method (HPM) ([4]-[10]). Prof. J. H. He first proposed this strategy in 1998 [8].

The purpose of this study is to study analytical and numerical solution of MHD natural convection of grade three of a non- Newtonian fluid flow between two vertical flat plates through a porous medium considering the effect heat source using Multi-step differential transform method and finite difference method. Furthermore, show how dimensionless non-Newtonian viscosity, porosity parameter, Hartman number, non-Darcy parameter, Eckert number, Prandtl number, and heat source parameter affect the velocity and temperature of flow between two infinite parallel vertical flat plates. (MDTM) and (FDM) are used to solve the reduced ordinary differential equations. This method offers highly accurate successive approximations of the solution. The velocity and temperature variation distributions, as well as the problem's governing parameters, are shown. Furthermore, graphical and numerical results for the velocity and temperature profiles are presented and discussed for various parametric conditions. Finally, comparisons with previously published works were made, revealing that the current results are highly accurate and in good agreement.

\section{Mathematical Formulation of the Problem}

Figure (1) depicts a diagram of the problem under investigation. It is made up of two vertically flat plates. Two flat plates separated by $2 \mathrm{~b}$ contain a non-Newtonian fluid. At $\mathrm{x}=$ $+b$ and $x=b$, the walls are kept at constant temperatures of $T_{1}$ and $T_{2}$, respectively, where $T_{1}$ $>\mathrm{T}_{2}$. The fluid near the wall at $\mathrm{x}=-\mathrm{b}$ rises, while the fluid near the wall at $\mathrm{x}=+\mathrm{b}$ falls, due to the temperature differential. Figure 1: Schematic diagram of the flow.

The motion equation is [20]:

$$
\mu \frac{d^{2} u}{d x^{2}}+6 \beta_{3}\left(\frac{d u}{d x}\right)^{2} \frac{d^{2} u}{d x^{2}}+\rho_{0} \gamma\left(\mathrm{T}-T_{m}\right) \mathrm{g}-\frac{\mu}{K} \mathrm{u}-\frac{\sigma \beta_{0}^{2}}{\rho} \mathrm{u}=0
$$

as well as the following energy equation:

$$
\kappa \frac{d^{2} T}{d x^{2}}+2 \beta_{3}\left(\frac{d u}{d x}\right)^{4}+\mu\left(\frac{d u}{d x}\right)^{2}+Q_{0}\left(\mathrm{~T}-T_{m}\right)=0,
$$

Using the similarity variables, Rajagopal [20] proved that:

$$
v=\frac{u}{u_{0}}, \eta=\frac{x}{b} \text { and } \theta=\frac{T-T_{m}}{T_{1}-T_{2}},
$$


The Navier-Stokes and energy equations can be simplified to a pair of ordinary differential equations as follows:

$$
\begin{aligned}
& \frac{d^{2} v}{d \eta^{2}}+6 \delta\left(\frac{d v}{d \eta}\right)^{2} \frac{d^{2} v}{d \eta^{2}}+\theta-P \mathrm{v}-H_{a}^{2} \mathrm{v}=0 \\
& \frac{d^{2} \theta}{d \eta^{2}}+2 \delta E_{c} P_{r}\left(\frac{d v}{d \eta}\right)^{4}+E_{c} P_{r}\left(\frac{d v}{d \eta}\right)^{2}+\alpha \theta=0
\end{aligned}
$$

$$
\text { Where } \delta=\frac{\beta_{3} u_{0}^{2}}{\mu b^{2}}, P=\frac{b^{2}}{K}, H_{a}^{2}=\frac{\sigma \beta_{0}^{2} b^{2}}{\rho \mu}, E_{c}=\frac{u_{0}^{2}}{c\left(T_{1-} T_{2}\right)}, P_{r}=\frac{\mu c}{\kappa} \text { and } \alpha=\frac{Q_{0} b^{2}}{\kappa}
$$

are dimensionless non-Newtonian viscosity, porosity parameter, Hartman number, Eckert number, Prandtl number and heat source parameter, respectively.

The appropriate boundary conditions are:

$$
\begin{aligned}
& \mathrm{v}=0, \theta=1 / 2 \text { at } \eta=-1, \\
& \mathrm{v}=0, \theta=-1 / 2 \text { at } \eta=1,
\end{aligned}
$$

\section{Analytical Method for Solution}

The reported findings were incorrect when the (DTM) was applied to solve differential equations with infinity boundary conditions or equations with very nonlinear behavior (when the boundarylayer variable goes to infinity, the resultant series solutions are divergent). Furthermore, power series are ineffective when the independent variable has high values. The (MDTM) has been designed for the analytical solution of differential equations to address this problem, and it is explained in this section. The following nonlinear initial value problem is considered for this purpose. The following recursive relations can be formed using the differential transformation of Eqs. (4) and (5):

$$
\begin{aligned}
& (\mathrm{k}+1)(\mathrm{k}+2) \mathrm{V}(\mathrm{k}+2)+6 \delta\left(r_{1}+1\right)\left(r_{2}-r_{1}+1\right)\left(\mathrm{k}-r_{2}+1\right)\left(\mathrm{k}-r_{2}+2\right) \\
& \mathrm{V}\left(r_{1}+1\right) \mathrm{V}\left(r_{2}-r_{1}+1\right) \mathrm{V}\left(\mathrm{k}-r_{2}+2\right)+\Theta(\mathrm{k})-\mathrm{PV}(\mathrm{k})-H_{a}^{2} \mathrm{~V}(\mathrm{k})=0, \\
& (\mathrm{k}+1)(\mathrm{k}+2) \Theta(\mathrm{k}+2)+E_{c} P_{r} \sum_{r=0}^{k}(r+1)(\mathrm{k}-\mathrm{r}+1) V(r+1) V(\mathrm{k}-r+1) \\
& +2 E_{c} P_{r} \sum_{r_{3=0}}^{k} \sum_{r_{2}=0}^{r_{3}} \sum_{r_{1}=0}^{r_{2}}\left(r_{1}+1\right)\left(r_{2}-r_{1}+1\right)\left(r_{3}-r_{2}+1\right) \\
& \left(\mathrm{k}-r_{3}+1\right) \mathrm{V}\left(r_{1}+1\right) \mathrm{V}\left(r_{2}-r_{1}+1\right) \mathrm{V}\left(r_{3}-r_{2}+1\right) V\left(\mathrm{k}-r_{3}+1\right)+\alpha \Theta(\mathrm{k})=0,
\end{aligned}
$$

Where the differential transforms of $\mathrm{u}(\eta)$ and $\theta(\eta)$ are $\mathrm{V}(\mathrm{k})$ and $\Theta(\mathrm{k})$, respectively.

The boundary conditions' differential transform (6-7) is as follows:

$$
\begin{aligned}
& \mathrm{V}(0)=0, \Theta(0)=\frac{1}{2}, \\
& \sum_{k=0}^{i} v(k) 2^{k}=0, \sum_{k=0}^{i} \Theta(k) 2^{k}=-\frac{1}{2},
\end{aligned}
$$


The following boundary conditions (6-7) can be considered:

$$
\begin{aligned}
& \mathrm{v}(-1)=0, \theta(-1)=\frac{1}{2}, \\
& \mathrm{v}^{`}(-1)=\lambda, \theta^{\prime}(-1)=\omega,
\end{aligned}
$$

The following is the differential transform of the above conditions:

$$
\begin{aligned}
& V(0)=0, \Theta(0)=\frac{1}{2}, \\
& V(1)=\lambda, \Theta(1)=\omega,
\end{aligned}
$$

We can also calculate alternative values of $\mathrm{V}(\mathrm{k})$ and $\Theta(\mathrm{K})$ by inserting equations (14) and (15) into equations (8) and (9) and using the recursive technique.

\section{Numerical Method for Solution}

FDM used to solve system of coupled nonlinear ordinary differential equations (4-5) with boundary conditions (6-7). Because of the nonlinearity of this system, the following linearized form should be used.:

$$
\begin{aligned}
& \frac{d^{2} v}{d \eta^{2}}\left(1+6 \delta\left(\frac{d \bar{v}}{d \eta}\right)^{2}\right)+\theta-\left(P+H_{a}^{2}\right) \mathrm{v}=0 \\
& \frac{d^{2} \theta}{d \eta^{2}}+E_{c} P_{r} \frac{d v}{d \eta}\left(2 \delta\left(\frac{d \bar{v}}{d \eta}\right)^{3}+\frac{d \bar{v}}{d \eta}\right)^{3}+\alpha \theta=0
\end{aligned}
$$

The iterated terms that transform the system of equations (4-5) into a linearized one are denoted by the bar notation. Taylor's expansions of the dependent variables about the central point are used to convert the linearized system of coupled non-linear ordinary differential equations (16-17) to system algebraic equations:

$$
\begin{gathered}
\frac{d v_{i}}{d \eta}=\frac{v_{i+1}-v_{i-1}}{\Delta}+\mathrm{o}\left(\Delta^{2}\right) \\
\frac{d^{2} v_{i}}{d \eta^{2}}=\frac{v_{i+1}-2 v_{i}+v_{i-1}}{\Delta^{2}}+\mathrm{o}\left(\Delta^{2}\right) \\
\frac{d^{2} \theta_{i}}{d \eta^{2}}=\frac{\theta_{i+1}-2 \theta_{i}+\theta_{i-1}}{\Delta^{2}}+\mathrm{o}\left(\Delta^{2}\right)
\end{gathered}
$$

Where $\mathrm{i}=1,2,3, \ldots \ldots, \mathrm{m}+1$ and $\mathrm{m}$ the number of subintervals of the finite domain of solution ($<\eta<1)$. 


\section{Results and Discussion}

(MDTM) and (FDM) effectively applied to address the problem of non-Newtonian fluid natural convection flow between two vertical flat plates. Tables and graphs of the results are very useful in showing the efficiency and accuracy of the (MDTM) and (FDM) for the problem presented in this paper. We compared the current results to previously published studies in order to ensure that they were accurate. The effects of Dimensionless non-Newtonian viscosity, porosity parameter $\mathrm{P}$, Hartman number $\mathrm{H}_{\mathrm{a}}$, Eckert number $\mathrm{E}_{\mathrm{c}}$, Prandtl number $\mathrm{P}_{\mathrm{r}}$, and heat source parameter $\boldsymbol{\alpha}$ on velocity V ( $\eta)$ and temperature $\theta(\eta)$ profiles are shown in Figures (2-7) (a) and (b).

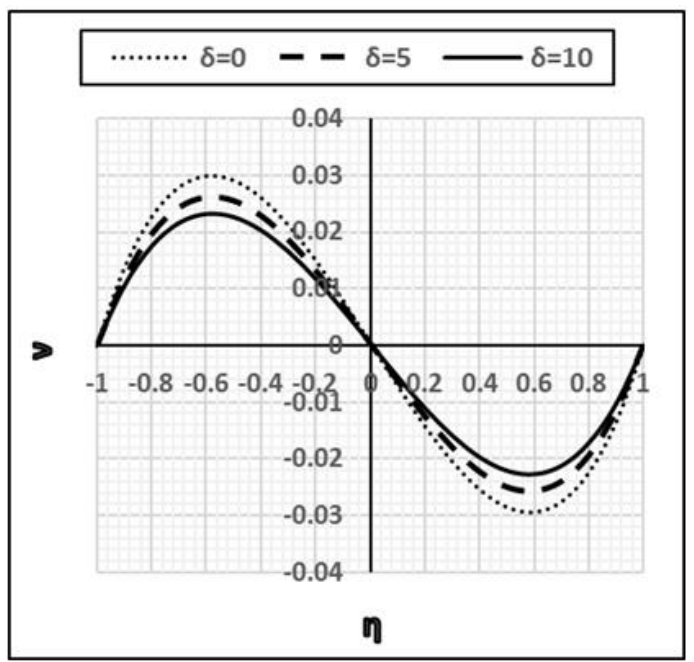

(a)

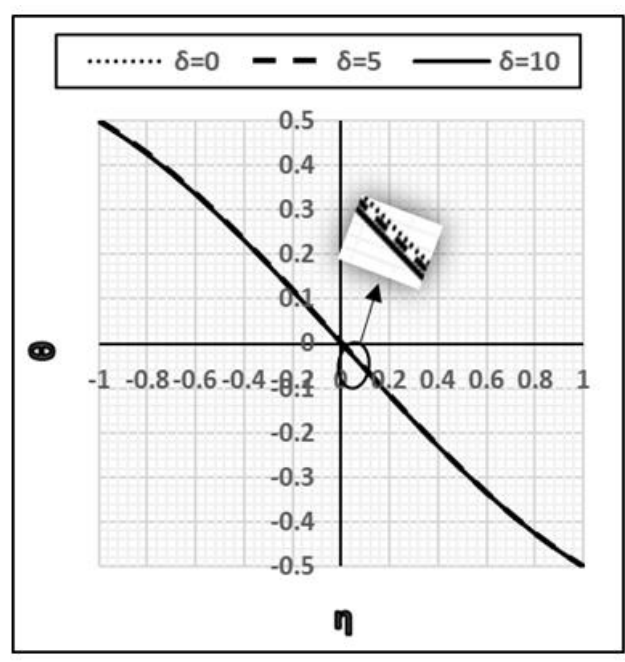

(b)

Figure 2: V $(\eta)$ and $\theta(\eta)$ results for a variety of $\delta$ when $\mathrm{P}=1, \mathrm{H}_{\mathrm{a}}=1, \mathrm{E}_{\mathrm{c}}=1, \mathrm{P}_{\mathrm{r}}=1$ and $\boldsymbol{\alpha}=1$.

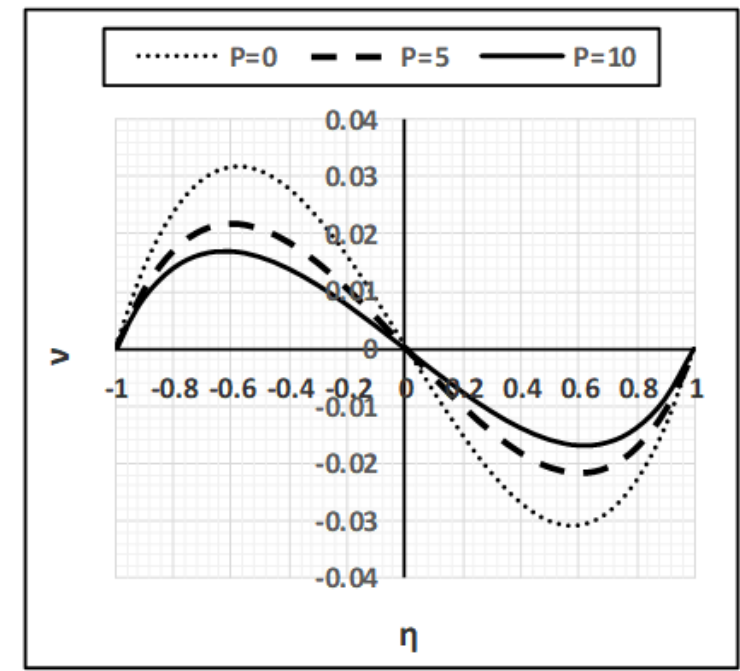

(a)

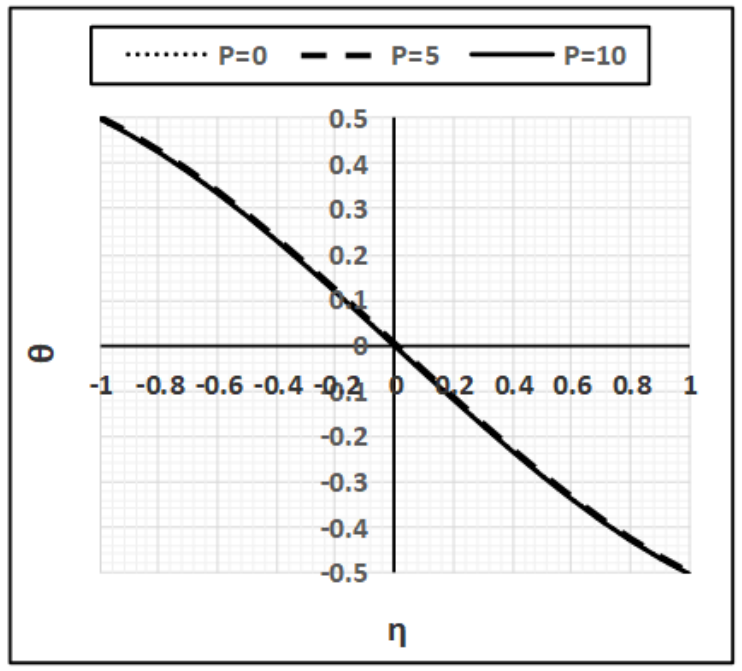

(b)

Figure 3: $V(\eta)$ and $\theta(\eta)$ results for a variety of $\mathrm{P}$ when $\delta=1, \mathrm{H}_{\mathrm{a}}=1, \mathrm{E}_{\mathrm{c}}=1, \mathrm{P}_{\mathrm{r}}=1$ and $\boldsymbol{\alpha}=1$.

It can be shown that increasing Dimensionless non-Newtonian viscosity, porosity parameter, and Hartman number causes to decrease velocity Figures (2-4) (a), but increasing Prandtl number, Eckert number, and heat source parameter causes to increase velocity Figures (5-7) (a). Also increases in the dimensionless non-Newtonian viscosity parameter result in temperature decreases 
in Figures (2)(b), but increases in the Prandtl number, Eckert number, and heat source parameter result in temperature increases in Figures (5-7) (b). Figures (3-4) (b) show effect of porosity parameter and Hartman number on temperature distribution is minimal, if not non-existent, because porosity parameter and Hartman number do not appear directly in energy equation. As a result, porosity parameter and Hartman number have little effect on the flow temperature.

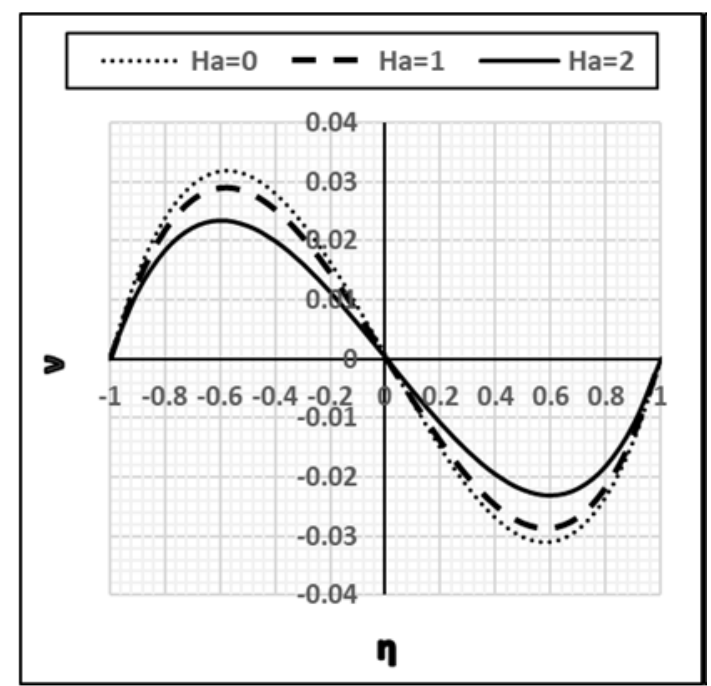

(a)

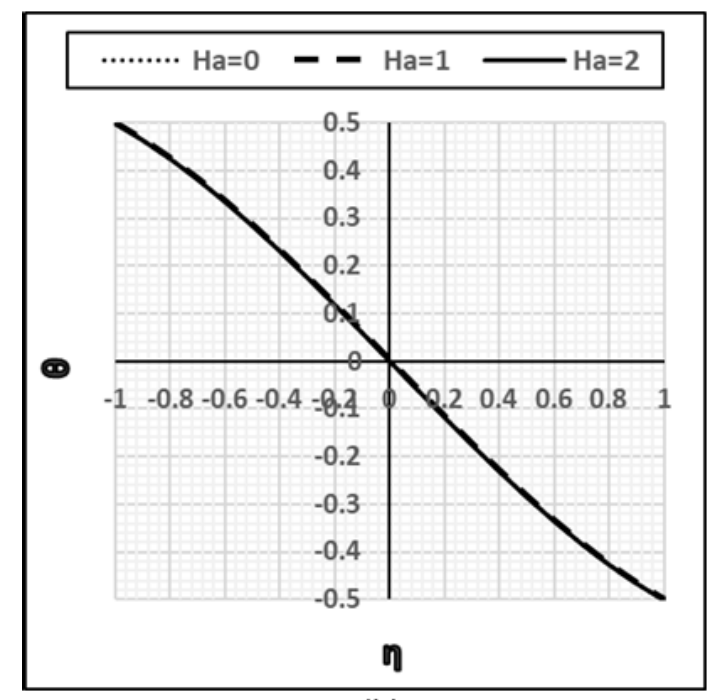

(b)

Figure 4: V ( $\eta)$ and $\theta(\eta)$ results for a variety of Ha when $\delta=1, \mathrm{P}=1, \mathrm{E}_{\mathrm{c}}=1, \mathrm{P}_{\mathrm{r}}=1$ and $\boldsymbol{\alpha}=1$.

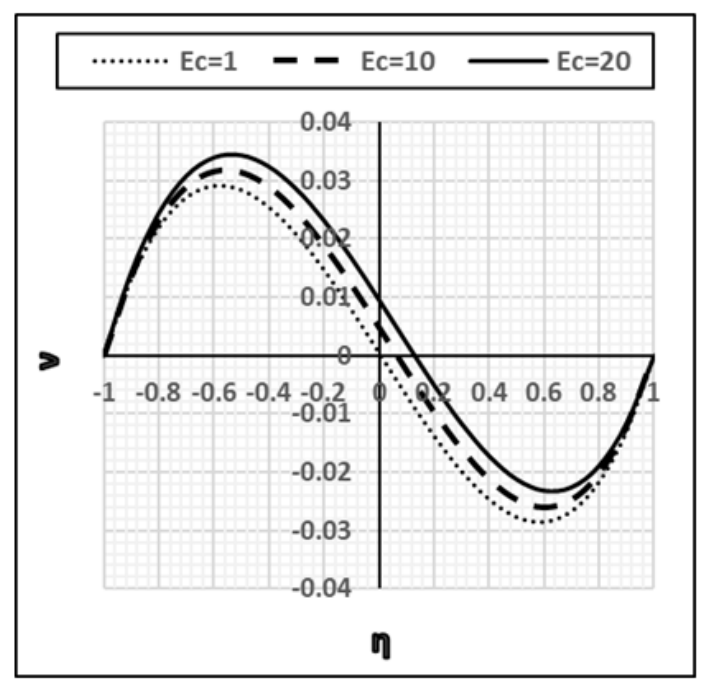

(a)

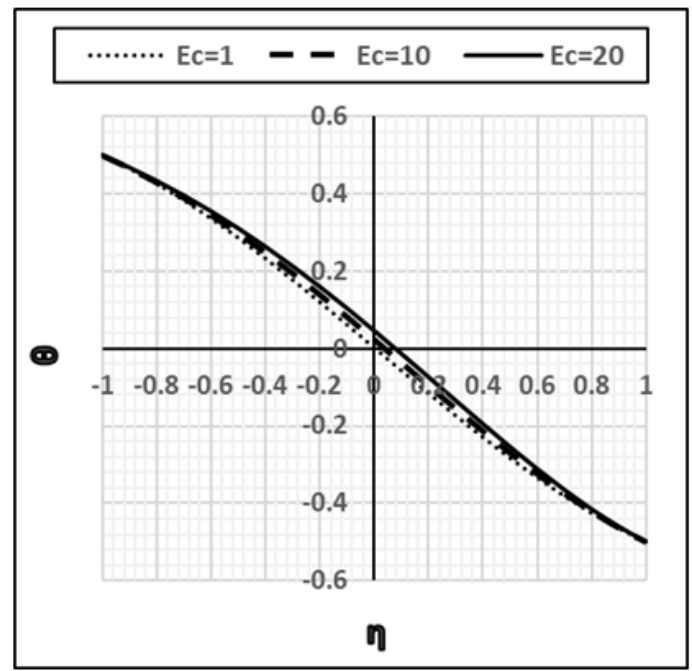

(b)

Figure 5: V ( $\eta)$ and $\theta(\eta)$ results for a variety of $\mathrm{E}_{\mathrm{c}}$ when $\delta=1, \mathrm{P}=1, \mathrm{H}_{\mathrm{a}}=1, \mathrm{P}_{\mathrm{r}}=1$ and $\boldsymbol{\alpha}=1$.

In addition, Tables (1-2) compare analytical and numerical solutions using (MDTM) and (FDM) with ((LSM) and (GM) [18])). This approximate analytical and numerical solution is found to be quite close to the equivalent solutions. 


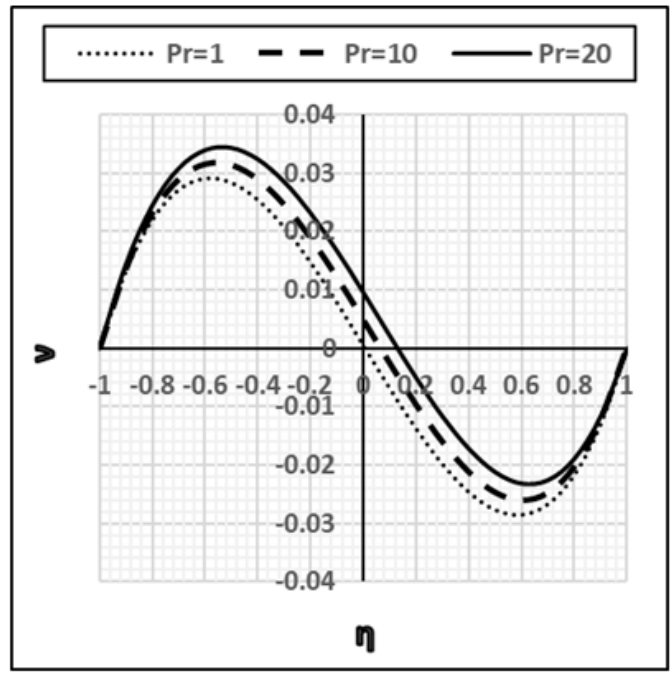

(a)

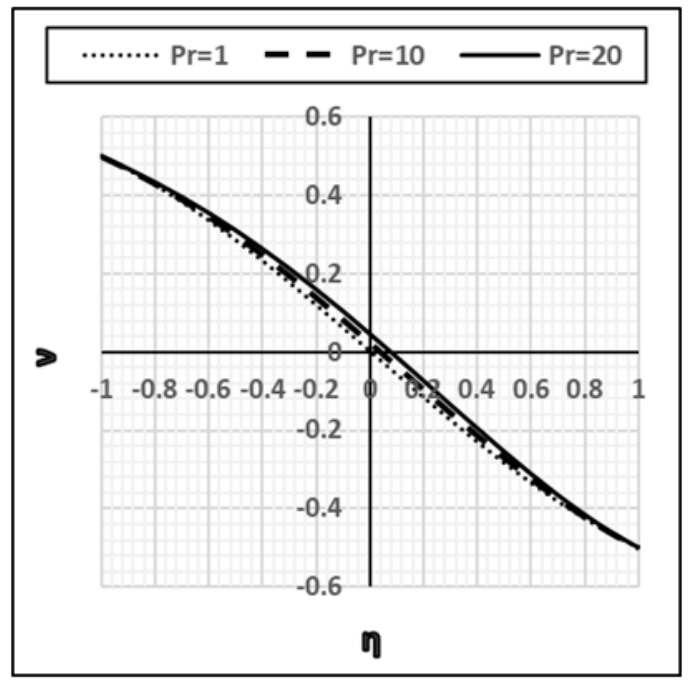

(b)

Figure 5: $V(\eta)$ and $\theta(\eta)$ results for a variety of $P_{\mathrm{r}}$ when $\delta=1, P=1, H_{a}=1, E_{c}=1$ and $\boldsymbol{\alpha}=1$.

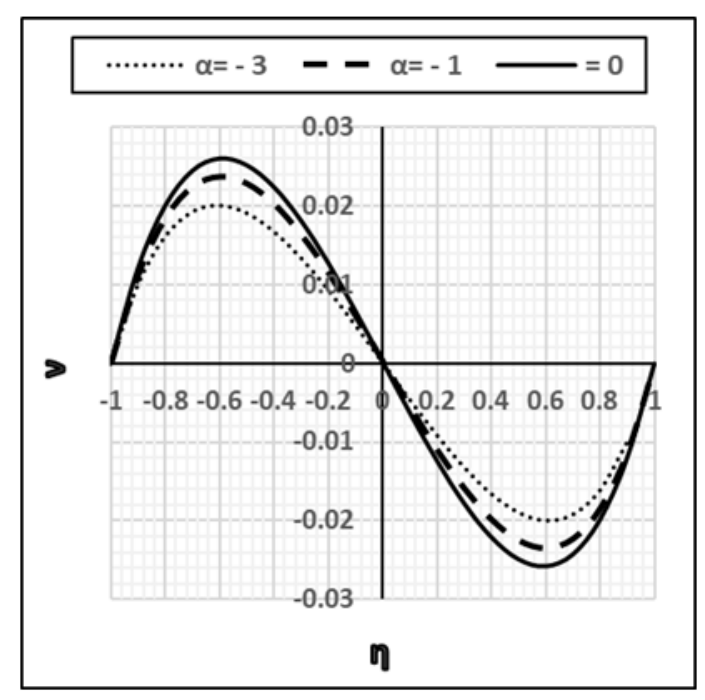

(a)

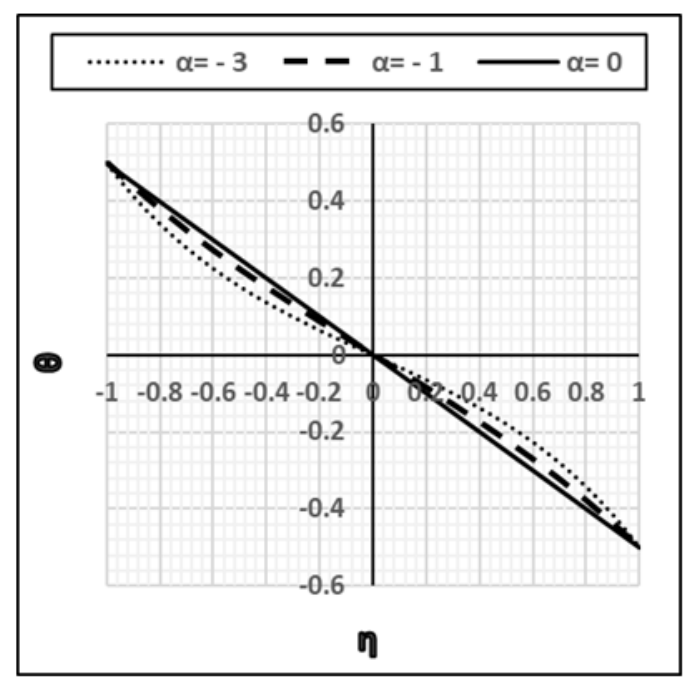

(b)

Figure 7: V ( $\eta$ ) and $\theta(\eta)$ results for a variety of $\alpha$ when $\delta=1, P=1, H_{a}=1, E_{c}=1$ and $\operatorname{Pr}=1$. 
Table 1: Comparison analytical and numerical solution by using (MDTM) and (FDM) with $((\mathrm{LSM})$ and $(\mathrm{GM})[18])$ solution for $\mathrm{V}(\eta)$ when $\delta=1, \mathrm{P}=1, \mathrm{Ha}=1, \mathrm{Ec}=1, \mathrm{Pr}=1$ and $\alpha=1$.

\begin{tabular}{|c||c|c|c|c|}
\hline \multicolumn{1}{|c||}{$\boldsymbol{\eta}$} & \multicolumn{2}{c|}{ Present V (n) } & \multicolumn{2}{c|}{$\mathbf{~}(\mathbf{\eta})[\mathbf{1 8}]$} \\
\cline { 2 - 5 } & MDTM & 0 & 0 & $\mathbf{G M}$ \\
\hline \hline-1 & 0 & 0.0130949483 & 0.01296 & 0.013011 \\
\hline-0.9 & 0.0131467243 & 0.0219453880 & 0.021828 & 0.021913 \\
\hline-0.8 & 0.0222041934 & 0.0270982482 & 0.027059 & 0.027163 \\
\hline-0.7 & 0.0275524420 & 0.0290697213 & 0.029106 & 0.029218 \\
\hline-0.6 & 0.0296391499 & 0.0283513261 & 0.028426 & 0.028534 \\
\hline-0.5 & 0.0289479137 & 0.0254150876 & 0.025473 & 0.025568 \\
\hline-0.4 & 0.0259710609 & 0.0207179808 & 0.0207 & 0.020775 \\
\hline-0.3 & 0.0211922398 & 0.0147057703 & 0.014563 & 0.014613 \\
\hline-0.2 & 0.0150787593 & 0.0078163715 & 0.007517 & 0.007538 \\
\hline-0.1 & 0.0080811050 & 0.0004828515 & $1.57 \mathrm{E}-05$ & $6.53 \mathrm{E}-06$ \\
\hline 0 & 0.0006368290 & -0.0068638171 & -0.00749 & -0.00753 \\
\hline 0.1 & -0.006823275 & -0.0137921405 & -0.01453 & -0.0146 \\
\hline 0.2 & -0.0138680651 & -0.0198675177 & -0.02067 & -0.02076 \\
\hline 0.3 & -0.0200589440 & -0.0246495768 & -0.02545 & -0.02556 \\
\hline 0.4 & -0.0249436890 & -0.0276893269 & -0.0284 & -0.02852 \\
\hline 0.5 & -0.0280525328 & -0.0285260120 & -0.02909 & -0.02921 \\
\hline 0.6 & -0.0288985481 & -0.0266835532 & -0.02704 & -0.02716 \\
\hline 0.7 & -0.0269851109 & -0.0216664577 & -0.02182 & -0.02191 \\
\hline 0.8 & -0.0218230424 & -0.0129550664 & -0.01295 & -0.01301 \\
\hline 0.9 & -0.01295756242 & 0 & 0 & 0 \\
\hline 1 & & & & \\
\hline
\end{tabular}


Table 2: Comparison analytical and numerical solution by using (MDTM) and(FDM) with ((LSM) and $(\mathrm{GM})[18])$ solution for $\theta(\eta)$ when $\delta=1, \mathrm{P}=1, \mathrm{Ha}=1, \mathrm{Ec}=1, \operatorname{Pr}=1$ and $\alpha=1$.

\begin{tabular}{|c|c|c|c|c|}
\hline \multirow{2}{*}{$n$} & \multicolumn{2}{|c|}{ Present $\theta(\eta)$} & \multicolumn{2}{|c|}{$\theta(\eta)[18]$} \\
\hline & MDTM & FDM & LSM & GM \\
\hline-1 & 0.5 & 0.5 & 0.5 & 0.5 \\
\hline-0.9 & 0.4660129153 & 0.4656457205 & 0.465147 & 0.465708161 \\
\hline-0.8 & 0.4272408396 & 0.4267202015 & 0.425513 & 0.426457054 \\
\hline-0.7 & 0.38414560967 & 0.3835818575 & 0.381631 & 0.382797641 \\
\hline-0.6 & 0.33719676278 & 0.3366353599 & 0.334031 & 0.335280886 \\
\hline-0.5 & 0.28687703651 & 0.2863273745 & 0.283244 & 0.28445775 \\
\hline-0.4 & 0.23368653860 & 0.2331419239 & 0.229801 & 0.230879199 \\
\hline-0.3 & 0.17814518812 & 0.1775954126 & 0.174232 & 0.175096193 \\
\hline-0.2 & 0.12079332678 & 0.1202313611 & 0.117069 & 0.117659697 \\
\hline-0.1 & 0.06219059768 & 0.0616148969 & 0.058842 & 0.059120673 \\
\hline 0 & 0.00291326870 & 0.0023270538 & 8.35E-05 & $3.01 \mathrm{E}-05$ \\
\hline 0.1 & -0.05644982195 & -0.0570410653 & -0.05868 & -0.059061105 \\
\hline 0.2 & -0.11530255996 & -0.1158942060 & -0.11691 & -0.117601934 \\
\hline 0.3 & -0.17304800480 & -0.1736389001 & -0.17408 & -0.175041439 \\
\hline 0.4 & -0.22909596887 & -0.2296893664 & -0.22966 & -0.230828656 \\
\hline 0.5 & -0.28287175376 & -0.2834733124 & -0.28312 & -0.284412623 \\
\hline 0.6 & -0.33382611181 & -0.3344375781 & -0.33392 & -0.335242377 \\
\hline 0.7 & -0.38144650428 & -0.3820535624 & -0.38155 & -0.382766955 \\
\hline 0.8 & -0.425269654243 & -0.4258223744 & -0.42545 & -0.426435393 \\
\hline 0.9 & -0.464895204894 & -0.4652796526 & -0.46511 & -0.465696729 \\
\hline 1 & -0.5 & -0.5 & -0.5 & -0.5 \\
\hline
\end{tabular}

\section{Conclusions}

In this work, (MDTM) and (FDM) used to compute MHD natural convection of grade three nonNewtonian fluid flow between two infinite parallel vertical plates through a porous medium, with the effect of heat source. As shown in figures and tables, results of (MDTM) and (FDM) are in good agreement with results of previous analytical solutions. Dimensionless non-Newtonian viscosity, porosity parameter, Hartman number, Eckert number, Prandtl number, and heat source parameter all have an effect on velocity and temperature, as shown in the figures. Increases in dimensionless nonNewtonian viscosity, porosity parameter, and Hartman number result in a decrease in velocity, but increases in Prandtl number, Eckert number, and heat source parameter result in an increase in velocity. It has also been discovered that increasing the dimensionless non-Newtonian viscosity parameter causes temperature to decreases, whilst increasing the Prandtl number, Eckert number, and heat source parameter causes temperature to increase. It can also be seen that the porosity parameter and the Hartman number have no effect on the flow temperature. Furthermore, comparisons with available previously published works are performed and showed that the present results have high accuracy and are found to be in good agreement as shown in tables. The results 
suggest that using (MDTM) and (FDM) to solve nonlinear differential equations like this one is a powerful approach.

\section{References}

[1] R.W. Bruce, T.Y. Na, Natural convection flow of Powell-Eyring fluids between two vertical flat plates, ASME67WA/HT-25, presented at the ASME Winter Annu Meet, Pittsburgh, Pennsylvania, (1967) $12-17$.

[2] Karlapalem, Vidyadhar, Subhasisa Rath, and Sukanta Kumar Dash. "Orientation effects on laminar natural convection heat transfer from branching-fins." International Journal of Thermal Sciences 142 (2019): 89-105.

[3] Liu, Jie, et al. "Laminar natural convection heat transfer from a pair of attached horizontal cylinders set in a vertical array." Applied Thermal Engineering 115 (2017): 1004-1019.

[4] GUPTA, Neelam; KANTH, Neel. Study of heat flow in a rod using homotopy analysis method and homotopy perturbation method. In: AIP Conference Proceedings. AIP Publishing LLC, (2019). p. 020013.

[5] Ghasemi, Seiyed E., Ali Zolfagharian, and D. D. Ganji. "Study on motion of rigid rod on a circular surface using MHPM." Propulsion and Power Research 3.3 (2014): 159-164.

[6] Khan, Yasir, and Habibolla Latifizadeh. "Application of new optimal homotopy perturbation and Adomian decomposition methods to the MHD non-Newtonian fluid flow over a stretching sheet." International Journal of Numerical Methods for Heat \& Fluid Flow (2014).

[7] Kharrat, Bachir Nour, and George Toma. "A New Hybrid Sumudu Transform with Homotopy Perturbation Method for Solving Boundary Value Problems." Middle-East Journal of Scientific Research 28.2 (2020): 142-149.

[8] J.H. He, an approximate solution technique depending on an artificial parameter, Communications in Nonlinear Science and Numerical Simulation, 3 (1998) 92-97.

[9] Khan, Yasir. "A series solution of the boundary value problem arising in the application of fluid mechanics." International Journal of Numerical Methods for Heat \& Fluid Flow (2018).

[10] Singh, Jagdeo, Devendra Kumar, and Dumitru Baleanu. "A hybrid analytical algorithm for thin film flow problem occurring in non-Newtonian fluid mechanics." Ain Shams Engineering Journal 12.2 (2021): 2297-2302.

[11] Turkyilmazoglu, Mustafa. "Accelerating the convergence of Adomian decomposition method (ADM)." Journal of Computational Science 31 (2019): 54-59.

[12] Turkyilmazoglu, Mustafa. "Parametrized adomian decomposition method with optimum convergence." ACM Transactions on Modeling and Computer Simulation (TOMACS) 27.4 (2017): 1-22.

[13] Abdelrahman, Mahmoud AE, Emad HM Zahran, and Mostafa MA Khater. "The Exp (- $\varphi(\xi)$ )-expansion method and its application for solving nonlinear evolution equations." International Journal of Modern Nonlinear Theory and Application 4.01 (2015): 37.

[14] Sadat, Rahma, and Magda Kassem. "Explicit solutions for the (2+ 1)-dimensional jaulent-miodek equation using the integrating factors method in an unbounded domain." Mathematical and Computational Applications 23.1 (2018): 15.

[15] S.H. Hosein Nia, H. Soltani, J. Ghasemi, A. N. Ranjbar, D. D. Ganji, Maintaining the stability of nonlinear differential equations by the enhancement of HPM, Phys Lett A, 372 (2008) 2855-2861.

[16] A. Kargar, M. Akbarzade, Analytic solution of natural convection flow of a non- Newtonian fluid between two vertical flat plates using homotopy perturbation method, International Journal of Engineering and Science, 1(3) (2012) 32-38. 
[17] Guo, Zhongjin, Lili Xia, and Wei Zhang. "Improving accurate vibration periods and responses of a rigid rod model." Alexandria Engineering Journal 57.3 (2018): 1331-1338.

[18] P. Maghsoudi, S. Sadeghi, H. Rasam, A. Amiri, Natural Convection of Third Grade Non-Newtonian Fluid Flow in a Changeable Semi- permeable Medium Considering the Effects of Variable Heat Source and Magnetic Field, European Journal of Sustainable Development,2(3) (2018).

[19] V. Marinca, N. Herisanu, Optimal homotopy perturbation method for stronglynonlinear differential equations, Nonlinear Science Letters A, 1 (2010) 273-280.

[20] K.R. Rajagopal, T.Y. Na, Natural convection flow of a non-Newtonian fluid between two vertical flat plates, Acta Mech, 54 (1985) 239-246.

[21] A.H. Nayfeh, Perturbation methods. Wiley, New York, (2000).

[22] Z. Ziabakhsh, G. Domairry, Analytic solution of natural convection flow of a non-Newtonian fluid between two vertical flat plates using homotopy analysis method, Commun Nonlinear Sci Numer Simulat, 14 (2009) 1868-1880.

[23] Nadeem, S., Rizwan Ul Haq, and Z. H. Khan. "Numerical solution of non-Newtonian nanofluid flow over a stretching sheet." Applied Nanoscience 4.5 (2014): 625-631. 